SOI: $1.1 /$ TAS $\quad$ DOI: $10.15863 /$ TAS International Scientific Journal Theoretical \& Applied Science

\author{
p-ISSN: 2308-4944 (print) e-ISSN: 2409-0085 (online) \\ Year: 2017 Issue: $07 \quad$ Volume: 51
}

Published: $30.07 .2017 \quad$ http://T-Science.org
Sergey Alexandrovich Mishchik Associate Professor, Candidate of Pedagogical Science, Academician of International Academy TAS, Assistant professor Department of Physics, State Maritime University Admiral Ushakov, Russia sergei_mishik@mail.ru

SECTION 21. Pedagogy. Psychology. Innovation in Education

\title{
SYSTEMIC PROBLEMS ATMOSPHERE ELECTRICITY OF APPLIED PHYSICS MARITIME FLOT OF PEDAGOGOMETRIC ANALYSIS
}

Abstract: The basic principles of the system of problems atmosphere electricity in applied physics Navy pedagogometric analysis of the formation of mathematical models of learning activities about the nature of achieving the criteria of life, cycling, systemsness and phasing, which form a basic cell of the educational space, as well as prima nenie twelve pointed star Ertsgammy relatively presentation ertsgamming principle which determines the foundations pedagogometric through forming substantive methods of hyper-space professional life, psychological and educational activity theory, psycho-pedagogical system analysis and the theory of the formation of mental actions.

Key words: pedagogometric, vital activity, cyclicity, system, phase, star Erzgammy, atmosphere electricity, applied physics, marine fleet.

Language: Russian

Citation: Mishchik SA (2017) SYSTEMIC PROBLEMS ATMOSPHERE ELECTRICITY OF APPLIED PHYSICS MARITIME FLOT OF PEDAGOGOMETRIC ANALYSIS. ISJ Theoretical \& Applied Science, 07 (51): 111-116.

Soi: http://s-o-i.org/1.1/TAS-07-51-19 Doi: crossef https://dx.doi.org/10.15863/TAS.2017.07.51.19

\section{УДК 372.851}

\section{СИСТЕМНЫЕ ЗАДАЧИ ЭЛЕКТРИЧЕСТВА АТМОСФЕРЫ ПРИКЛАДНОЙ ФИЗИКИ МОРСКОГО ФЛОТА ПЕДАГОГОМЕТРИЧЕСКОГО АНАЛИЗА}

Аннотация: Рассмотрены основные принципы построения системных задач электричества атмосферы прикладной физики морского флота педагогометрического анализа при формировании математических моделей учебной деятельности относительно характера достижения критериев жизнедеятельности, цикличности, системности и этапности, которые образуют базисную ячейку образовательного пространства, а также применение двенадцати конечной звезды Эригаммы относительно представления приниипа эригаммности, который определит основы педагогометрики через формообразование предметными методами гиперпространства профессиональной жизнедеятельности, психолого-педагогической теории деятельности, психолого-педагогического системного анализа и теории формирования умственных действий.

Ключевые слова: педагогометрика, жизнедеятельность, иикличность, системность, этапность, звезда Эригаммы, электричество атмосферы, прикладная физика, морской флот.

\section{Introduction}

Формирование системных задач электричества атмосферы прикладной физики морского флота педагогометрического анализа связывается с решением проблемы формирования математических моделей учебной деятельности через базисные представления методологии педагогометрического анализа, который отражает особенности структуры и жизнедеятельности, цикличности, системности и этапности. Результатом педагогометрического анализа является формирование базисной ячейки образовательного пространства, которая отражает принцип эрцгаммности относительно структуру двенадцати конечной звезды Эрцгаммы. Полученнная зависимость устанавливает основы педагогометрики через представление предметных методов гиперпространства 
профессиональной

жизнедеятельности, психолого-педагогической теории деятельности, психолого-педагогического системного анализа и теории формирования умственных действий $[1,2,3]$.

Установленные представления направлены на совершенствование базы предметных прикладных профессиональных задач электричества атмосферы прикладной физики морского флота педагогометрического анализа, отражающих целостную профессиональную деятельность на морском флоте. Формирование математических моделей учебнопрофессиональной деятельности связываются с: базисной звездой Эрцгаммы гиперпространства жизнедеятельности (Е1); базисным целостносистемным циклом жизнедеятельности (Е2); базисной звездой Эрцгаммы системного анализа (E3); базисным проявлением двенадцати этапов и форм познавательного гиперпространства жизнедеятельности относительно образовательного процесса (E4) $[4,5,6]$.

Адаптивное проектирование системных задач электричества атмосферы прикладной физики морского флота педагогометрического анализа и заданной базы предметных педагогометрических моделей эрцгаммного анализа образовательных объектов с признаком базисно-нормативной эрцгаммности, отражают их инвариантные структуры. При этом выполняется собственная функция психологоматематического представления профессионально-значимых объектов системных задач электричества атмосферы прикладной физики морского флота педагогометрического анализа через единство признаков смыслообразования учебно-профессионального действия, его принятия, ориентировочноисполнительно-контрольных признаков и прогноза совершенствования анализа объектов педагогометрческого содержания [7,8,9].

\section{Materials and Methods}

Системные задачи электричества атмосферы прикладной физики морского флота педагогометрического анализа отражают целостно-системное моделирование основных элементов транспортных объектов. При этом возникает ориентация на единство базисных характеристик предметных и исполнительных условий относительно предмета содержания и способа его реализации через представление базисной ячейки образовательного пространства, которая отражает принцип эрцгаммности адекватного структуре двенадцати конечной звезды Эрцгаммы.. Рассматриваются: ионизация атмосферы; характеристики ионизации атмосферного воздуха; ионизаторы атмосферного воздуха; ионосфера и полярные сияния; электрическое поле и токи в атмосфере; характеристики электрического поля тропосферы, применяемые в гидрометеорологических задачах на морском флоте.

В процессе решения системных задач электричества атмосферы прикладной физики морского флота педагогометрического анализа необходимо применять основные положения теории деятельности, системного анализа и теории формирования интеллекта через построение математических моделей учебнопрофессиональной активности отражающей структуру: базисной звездой Эрцгаммы гиперпространства жизнедеятельности (E1); базисного целостно-системного циклом жизнедеятельности $(\mathrm{E} 2)$; базисной звездой Эрцгаммы системного анализа (Е3); базисного проявления двенадцати этапов и форм познавательного гиперпространства жизнедеятельности относительно образовательного процесса (Е4).

Системный анализ предполагает выполнение последовательности системных аналитических действий: выделить объект анализа -задачу электричества атмосферы прикладной физики морского флота (ЗЭАПФМФ) как систему; установить порождающую среду ЗЭАПФМФ; определить уровни анализа ЗЭАПФМФ; представить целостные свойства ЗЭАПФМФ относительно пространственных, и временных характеристик и их комбинаций; выделить структуру уровня анализа ЗЭАПФМФ; установить структурные элементы уровня анализа ЗЭАПФМФ; определить системообразующие связи данного уровня анализа ЗЭАПФМФ; представить межуровневые связи анализа ЗЭАПФМФ; выделить форму организации ЗЭАПФМФ; установить системные свойства и поведение ЗЭАПФМФ.

\section{Задача 1}

В $1 \mathrm{~m}^{3}$ воздуха над океаном содержится $8 \cdot 10^{8}$ пар легких ионов. Сколько пар ионов в секунду должны создавать ионизаторы в $1 \mathrm{~m}^{3}$, чтобы при наличии только рекомбинации легких ионов друг с другом поддерживалось бы стационарное состояние? Коэффициент рекомбинации в чистом воздухе составляет $1,6 \cdot 10^{-12} \mathrm{~m}^{3} / \mathrm{c}$. Сколько пар ионов рекомбинирует каждую секунду в $1 \mathrm{~m}^{3}$ ? Если число ионов будет больше $8 \cdot 10^{8}$ (меньше $8 \cdot 10^{8}$ ) пар ионов $/ \mathrm{M}^{3}$, то должны ли ионизаторы для поддержания стационарного состояния создавать большее или меньшее число пар ионов? Как изменяется скорость рекомбинации легких ионов при возрастании числа ионов, при уменьшении числа ионов?

Ответ: $10^{6}$ пар ионов рекомбинирует каждую секунду в 1 м $^{3}$ - 


\begin{tabular}{|c|c|c|c|c|c|c|}
\hline Impact Factor: & $\begin{array}{l}\text { ISRA (India) } \\
\text { ISI (Dubai, UAE } \\
\text { GIF (Australia) } \\
\text { JIF }\end{array}$ & $\begin{array}{l}=1.344 \\
=0.829 \\
=0.564 \\
=1.500\end{array}$ & $\begin{array}{l}\text { SIS (USA) } \\
\text { PИНЦ (Russia) } \\
\text { ESJI (KZ) } \\
\text { SJIF (Morocco) }\end{array}$ & $\begin{array}{l}=0.912 \\
=0.234 \\
=\mathbf{3 . 8 6 0} \\
=\mathbf{2 . 0 3 1}\end{array}$ & $\begin{array}{l}\text { ICV (Poland) } \\
\text { PIF (India) } \\
\text { IBI (India) }\end{array}$ & $\begin{array}{l}=6.630 \\
=1.940 \\
=4.260\end{array}$ \\
\hline
\end{tabular}
$\left.\mathbf{M}^{3}\right)$.

$\left[10^{6}\right.$ пар ионов/(с• м $\left.\left.{ }^{3}\right)\right] ; 10^{6}$ пар ионов/(с•

\section{Задача 2}

Вычислить скорость движения легких ионов при средней напряженности электрического поля у поверхности Земли 130 B/м. Сравнить со скоростью вертикальных движений при тепловой и динамической конвекции. Средние значения подвижностей ионов $\omega_{+}=1,35 \cdot 10^{-4} \mathrm{M}^{2} /(\mathrm{B} \cdot \mathrm{c})$ и $\omega=$ $1,83 \cdot 10^{-4} \mathrm{M}^{2} /(\mathrm{B} \cdot \mathrm{c})$.

Ответ: $v_{1}=1,76 \cdot 10^{-2} \mathrm{M} /$ с и $v_{2}=2,38 \cdot 10^{-2} \mathrm{M} / \mathrm{c}$.

\section{Задача 3}

По теории Ланжевена подвижность иона обратно пропорциональна его массе. Во сколько раз будут различаться скорости движения иона радиусом $10^{-8}$ м и облачной капли радиусом 4 мкм в электрическом поле?

Ответ: в $1,6 \cdot 10^{4}$ раз.

\section{Задача 4}

Над океаном в 1 м $^{3}$ воздуха содержится $8,5 \cdot 10^{8} \quad \mathrm{M}^{-3} \quad$ положительных и $7 \cdot 10^{8} \quad \mathrm{M}^{-3}$ отрицательных легких ионов. Их средние подвижности равны соответственно $1,4 \cdot 10^{-4}$ $\mathrm{M}^{2} /(\mathrm{B} \cdot \mathrm{c})$ и $1,6 \cdot 10^{-4} \mathrm{M}^{2} /(\mathrm{B} \cdot \mathrm{c})$. Определить: полярные проводимости, общую проводимость воздуха, удельное сопротивление воздуха над океаном. Во сколько раз воздух как изолятор хуже янтаря, если удельное сопротивление янтаря $5 \cdot 10^{14}$ см'м. Почему над поверхностью океана больше положительных ионов, чем отрицательных?

Ответ: $1,9 \cdot 10^{-14}, 1,8 \cdot 10^{-14}$ и $3,7 \cdot 10^{-14} \mathrm{OM}^{-1} \cdot \mathrm{M}^{-}$ ${ }^{1} ; 2,7 \cdot 10^{13}$ Ом'м ; в 18,5 раз.

\section{Задача 5}

Подвижность ионов зависит от атмосферного давления. Оцените изменение подвижности ионов и проводимости воздуха при замене циклона с давлением в центре $\boldsymbol{P}_{\boldsymbol{1}}=950$ мбар на антициклон с давлением $\boldsymbol{P}_{2}=1070$ мбар.

Ответ: уменьшатся на $11,2 \%$.

\section{Задача 6}

Проводимость воздуха определялась аспирационным методом. Внутренний электрод цилиндрического конденсатора был заряжен отрицательно до потенциала 140 В. Вентилятор просасывал воздух в течение 10 мин и потенциал уменьшился до 57 В. Вычислить полярную проводимость и коэффициент рассеяния отрицательного заряда, если емкости цилиндрического конденсатора и электрометра равны соответственно $1,4 \cdot 10^{-11}$ и $0,6 \cdot 10^{-11} \Phi$.

См/м.

Ответ: $\lambda=3,53 \cdot 10^{-15} \mathrm{CM} / \mathrm{M} ; \quad a=4,47 \cdot 10^{-14}$

\section{Задача 7}

Электропроводность воздуха можно представить как функцию высоты в виде: $\lambda(z)=2,72 \cdot 10^{-14} \exp \left(\frac{z-6}{3,5}\right)$, где (z- высота в км). Определить электропроводность воздуха на высоте 40 км. Сравнить полученную величину с электропроводностью морской воды, равной 3,3 $\mathrm{OM}^{-1} \cdot \mathrm{M}^{-1}$.

Ответ: $\lambda=4,48 \cdot 10^{-10} \mathrm{Oм}^{-1} \cdot \mathbf{M}^{-1}$, меньше чем у морской воды $7,4 \cdot 10^{9}$ раза.

\section{Задача 8}

Вычислить продолжительность жизни $\boldsymbol{\tau}$ легкого иона в запыленном воздухе акватории порта, где постоянная исчезновения легких ионов $\boldsymbol{\beta}$ принимает предельное значение $30 \cdot 10^{-9} \mathrm{~m}^{3} / \mathrm{c}$. Определить диапазон изменений $\boldsymbol{\tau}$ легкого иона в зависимости от степени загрязнения воздуха акватории порта, если $\boldsymbol{\beta}$ изменяется в пределах $(1-30) \cdot 10^{-9} \mathrm{M}^{3} / \mathrm{c}$ ?

$$
\text { Ответ: } \tau=8.7 .33 \mathrm{c} ; \tau=[33 \mathrm{c} ; 16 \text { мин] }
$$

\section{Задача 9}

По теории Ланжевена подвижность ионов пропорциональна отношению заряда к массе. Определить подвижность электрона, если при тех же условиях подвижность легкого иона, состоящего из 10 молекул воды, равна $\omega_{\varepsilon}=1,35$ $\mathrm{cm}^{2} /(\mathrm{B} \cdot \mathrm{c}) ?$

Ответ: $\omega=3,0 \cdot 10^{5} \mathrm{~cm}^{2} /(\mathrm{B} \cdot \mathrm{c})$.

\section{Задача 10}

Определить время релаксации электрического состояния атмосферы, если концентрация ионов обоих знаков равна $\boldsymbol{n}_{+/-}=300$ $\mathrm{cm}^{-3}$ и их подвижность - зависимость между скоростью ионов и напряжённость поля - $\omega_{\boldsymbol{s}}=2$ $\mathrm{cm}^{2} /(\mathrm{B} \cdot \mathrm{c})$ ?

$$
\text { Ответ: } T_{p е л}=460 \mathrm{c} \text {. }
$$

\section{Задача 11}

Космические лучи создают у поверхности Земли в среднем около $\boldsymbol{N}_{\boldsymbol{I}}=1,8 \cdot 10^{6}$ пар ионов/(c· $\left.{ }^{3}\right)$, а радиоактивные ионизаторы, действующие практически только над сушей, создают около $\boldsymbol{N}_{2}=9 \cdot 10^{6}$ пар ионов $\left(\mathrm{c}^{\cdot} \mathrm{M}^{3}\right)$. Определить концентрацию легких ионов над океаном и над сушей, если бы воздух был идеально чистым. Почему в действительности число легких ионов над океаном имеет такой же порядок, как над сушей, хотя интенсивность ионообразования над сушей почти в 5 раз больше, чем над океаном.

Ответ: $n_{1}=1 \cdot 10^{9} ; n_{2}=2,5 \cdot 10^{9}$ пар ионов $/ \mathrm{M}^{3}$.

\section{Задача 12}

Определить реальные коэффициенты исчезновения легких ионов над океаном и над сушей, если над океаном в среднем наблюдается 
около $\boldsymbol{n}_{\boldsymbol{l}}=6 \cdot 10^{8}$ пар ионов $/ \mathrm{M}^{3}$, а над сушей около $\boldsymbol{n}_{2}=7 \cdot 10^{8}$ пар ионов $/ \mathrm{M}^{3}$. Космические лучи создают у поверхности Земли в среднем около $\boldsymbol{N}_{\boldsymbol{I}}$ $=1,8 \cdot 10^{6}$ пар ионов $/\left(\mathrm{c}^{*} \mathrm{M}^{3}\right)$, а радиоактивные ионизаторы, действующие практически только над сушей, создают около $\boldsymbol{N}_{2}=9 \cdot 10^{6}$ пар ионов $\left(\mathrm{c}^{\cdot} \mathrm{M}^{3}\right)$. Во сколько раз и почему скорость исчезновения легких ионов над сушей больше, чем над океаном?

$$
\text { Ответ: } k_{1}=3 \cdot 10^{-3} \mathrm{c}^{-1} ; \quad k_{2}=14 \cdot 10^{-3} \mathrm{c}^{-1} \text {. }
$$

\section{Задача 13}

Суточные изменения градиента потенциала электрического поля атмосферы над всеми океанами происходят одновременно (унитарная вариация). Максимум наблюдается около 17-18 ч, а минимум - около $3-4$ ч по Гринвичу. Средний годовой максимум и минимум составляют соответственно 165 В/м и 115 B/м. Вычислить поверхностные плотности заряда в момент наступления максимума и минимума напряженности электрического поля.

Ответ: $\sigma_{1}=-1,45 \cdot 10^{-9} \mathrm{Kл} / \mathbf{M}^{2} ; \sigma_{2}=$ $1,01 \cdot 10^{-9}$ Кл/ $/ \mathbf{M}^{2}$.

\section{Задача 14}

Вычислить плотность тока, который обеспечивает такое изменение поверх-ностного заряда океана, и силу тока, проходящего ко всей поверхности Мирового океана, если его площадь равна $\boldsymbol{S}=3,61 \cdot 10^{8}$ км $^{2}$. Суточные изменения градиента потенциала электрического поля атмосферы над всеми океанами происходят одновременно (унитарная вариация). Максимум наблюдается около 17-18 ч, а минимум - около 3-4 ч по Гринвичу. Средний годовой максимум и минимум составляют соответственно 165 В/м и 115 В/м. Поверхностные плотности заряда в момент наступления максимума и минимума напряженности электрического поля равны $\boldsymbol{\sigma}_{\boldsymbol{l}}=$ $1,45 \cdot 10$ - 9 Кл/ $/ \mathrm{M}^{2} ; \sigma_{2}=-1,01 \cdot 10-9$ Кл/ $\mathrm{M}^{2}$.

Ответ: $J=\left[0,87 \cdot 10^{-14} \mathrm{~A} / \mathrm{M}^{2} ; 1,2 \cdot 10^{-14} \mathrm{~A} / \mathrm{M}^{2}\right]$; $I=[3,1 \mathrm{~A} ; 4,3 \mathrm{~A}]$.

\section{Задача 15}

Определить напряженность электрического поля, которую создает у поверхности мирового океана Земли грозовое облако $\boldsymbol{C b}$ под облаком и на расстоянии $\boldsymbol{r}=20$ км, если центр тяжести отрицательного заряда облака находится на высоте $\boldsymbol{h}=3$ км, а заряд равен $\boldsymbol{q}=20$ Кл. На каком расстоянии поле, создаваемое облаком, становится сравнимым с нормальным электрическим полем атмосферы?

Ответ: $E_{1}=40000 \mathrm{~B} / \mathrm{M} ; E_{2}=130 \mathrm{~B} / \mathrm{M} ; r_{2}=$ 20 км.

Задача 16

Определить максимальное расстояние, на котором можно обнаружить приближающееся грозовое облако над океаном прибором для измерения напряженности электрического поля, если его чувствительность равна $\boldsymbol{E}=1$ В/м? Заряд нижней части грозового облака $\boldsymbol{C b}$ равен $\boldsymbol{q}=30$ Кл, центр тяжести заряда расположен на высоте $\boldsymbol{h}$ $=2$ км.

$$
\text { Ответ: } r=100 \kappa м \text {. }
$$

\section{Задача 17}

Облачная морская капля радиусом $\boldsymbol{r}=10$ мкм имеет заряд $\boldsymbol{q}=500 \boldsymbol{e}$, где $\boldsymbol{e}=1,6 \cdot 10^{-19}$ Кл, и находится в вертикальном электрическом поле напряженностью $\boldsymbol{E}=150 \mathrm{~B} / \mathrm{M}$. Определить соотношение между силой тяжести и силой Кулона, действующей на облачную морскую каплю.

Ответ: сила тяжести в 3400 раз больше силы Кулона.

\section{Задача 18}

Подсчитать среднюю плотность $\rho$ электрических зарядов в атмосфере, если известно, что напряженность электрического поля над поверхностью океана равна $100 \mathrm{~B} / \mathrm{M}$, а на высоте $\mathrm{h}=1,5$ км эта напряженность падает до $25 \mathrm{~B} / \mathrm{M}$.

Ответ: плотность $\rho$ электрических зарядов в атмосфере примерно равна $-1,3 \times 10^{-12}$ Кл $\bullet$ м-3.

\section{Задача 19}

Оценить количество молекул воздуха в атмосфере Земли при её абсолютной ионизации.

Ответ: $\cong 10^{44}$ молекул воздуха

\section{Conclusion}

Процесс формирования широкопрофильного профессионального мышления ориентируется на организацию всестороннего развития педагогометрической эрцгаммности. Представленные системные задачи электричества атмосферы прикладной физики морского флота педагогометрического анализа выражают основные направления развития и совершенствования базы прикладных предметных педагогометрических моделей образовательных объектов относительно педагогометрического математического моделирования учебного процесса. Это связывается с процессами совершенствования программируемых математических моделей учебной деятельности относительно характера достижения критериев жизнедеятельности, цикличности, системности и этапности.

Педагогометрический математический анализа позволяет применять линейную регрессию и корреляцию в педагометрических процессах, определять смысл и оценку параметров; проводить оценку значимости 
параметров линейной регрессии и корреляции; проводить интервальный прогноз на основе линейного уравнения регрессии; подбирать линеаризующие преобразования; отбирать факторы регрессии; выбирать формы уравнения регрессии; оценивать параметры уравнения множественной регрессии; частные уравнения регрессии; множественную и частную корреляцию; оценивать надежности результатов множественной регрессии и корреля-ции; вводить фиктивные переменные во множественной регрессии; применять обобщенный метод наименьших квадратов; метод максимального правдоподобия; исследовать основные элементы временного ряда и автокорреляцию уровней временного ряда; выявлять его структуру; моделировать тенденции временного ряда; моделировать особенные и циклических колебания; строить. аддитивную модель временного ряда и мультипликативную модель; применять фиктивные переменные для моделирования особенных колебаний.

Процесс формирования вектора педагогометрического математического моделирования учебного процесса связывается с установлением критериев эффективного функционирования образовательной системы относительно качества, как многомерной эрцгаммной совокупности. При этом текущее состояние образовательного процесса определяется конечным набором определенных числовых показателей X1, X2, X3... Xn,. Целью управления образовательной системы педагогометрического анализа является приведение текущего показателя уровня подготовки широкопрофильно-инновационных специалистов к максимальному показателю. При применении в педагогометрическом математическом моделировании учебного процесса теории графов выделяются правила формирования моделей: ни один учебный элемент модели не может быть представлен в виде нулевого графа; вершина графа, не являющаяся исходной, должна иметь минимум две нисходящих связи; в качестве исходных вершин графа могут применяться уже усвоенные учебные элементы.

Выделенные процессы образуют базисную ячейку образовательного пространства, а также выражают смысл двенадцати конечной звезды Эрцгаммы относительно представления принципа эрцгаммности. Представленные структуры устанавливают основы педагогометрики через формообразование предметными методами гиперпространства профессиональной жизнедеятельности, психолого-педагогической теории деятельности, психолого-педагогического системного анализа и теории формирования умственных действий. Установленные критерии жизнедеятельности, цикличности, системности и этапности, которые образуют базисную ячейку образовательного пространства, порождают условия формирования абсолютного инновационного образовательного цикла, отражающего специфическую структуру подготовки широкопрофильно-инновационных специалистов при реализации международных образовательных стандартов эревнометрического содержания алигорамной формы.

\section{References:}

1. Mishchik SA (2014) Pedagogometrika and mathematical modeling educational activity. Materialy Mezhdunarodnoy nauchnoy konferenctsii "Modern mathematics in science" - 30.06.2014. ISJ Theoretical \&Applied Science 6(14): 54-56 Caracas, Venezuela. doi: http://dx.doi.org/10.15863/TAS.2014.06.14.10

2. Mishchik SA (2014) Simulation training activity methods of mathematical logic. Materialy Mezhdunarodnoy nauchnoy konferenctsii "Eurapean Science and Education" - 30.07.2014. ISJ Theoretical \&Applied Science 6(15): 72-74 Marseille,
France.

doi: http://dx.doi.org/10.15863/TAS.2014.07.15.13

3. Mishchik SA (2014) Mathematical modeling system integrity-cycle of life activity - first goal pedagogometriki. Materialy Mezhdunarodnoy nauchnoy konferenctsii "European Applied Sciences" - 30.08.2014. ISJ Theoretical \&Applied Science 7(16): 77-79. Aix-en-Provence, France. doi: http://dx.doi.org/10.15863/TAS.2014.08.16.13

4. Mishchik SA (2014) Mathematical modeling system integrity-curricular activities - the second problem pedagogometriki. Materialy 


\begin{tabular}{l|lrl|l|ll} 
& ISRA (India) & $=\mathbf{1 . 3 4 4}$ & SIS (USA) & $=\mathbf{0 . 9 1 2}$ & ICV (Poland) & $=\mathbf{6 . 6 3 0}$ \\
Impact Factor: & ISI (Dubai, UAE) $=\mathbf{0 . 8 2 9}$ & PVHIL (Russia) $=\mathbf{0 . 2 3 4}$ & PIF (India) & $=\mathbf{1 . 9 4 0}$ \\
& GIF (Australia) & $\mathbf{0 . 5 6 4}$ & ESJI (KZ) & $=3.860$ & IBI (India) & $=\mathbf{4 . 2 6 0}$
\end{tabular}

Mezhdunarodnoy nauchnoy konferenctsii "European Innovation" - 30.09.2014. ISJ Theoretical \&Applied Science 9(17): 126-128 Martigues, France. doi: http://dx.doi.org/10.15863/TAS.2014.09.17.21

5. Mishchik SA (2014) Mathematical modeling holistic-systemic communicative activity - the third task pedagogometriki. Materialy Mezhdunarodnoy nauchnoy konferenctsii "European Scientific Achievements" 30.10.2014. ISJ Theoretical \&Applied Science 10(18): 45-47 Brighton, UK. doi: http://dx.doi.org/10.15863/TAS.2014.10.18.11

6. Mishchik SA (2014) Mathematical modeling integrity - system performance subject - fourth task pedagogometriki. Materialy Mezhdunarodnoy nauchnoy konferenctsii "Eurapean Science and Technology" 30.11.2014. ISJ Theoretical \&Applied Science 11(19): 51-54 Southampton, UK. doi: http://dx.doi.org/10.15863/TAS.2014.11.19.10

7. Mishchik SA (2015) Pedagogometrik - science and academic subject. Materialy Mezhdunarodnoy nauchnoy konferenctsii "European Technology in Science" 28.02.2015. ISJ Theoretical \& Applied Science 02 (22): 103-106 Malmö, Sweden. doi: http://dx.doi.org/10.15863/TAS.2015.02.22.17

8. Zvereva S. V. (1980) Zadachnik po obshhej meteorologii. L.: Gidrometeoizdat - p.124

9. Tokmazov GV (2014) Matematicheskoe modelirovanie v uchebno-professional'noy deyatel'nosti. Materialy Mezhdunarodnoy nauchnoy konferentsii «Modern mathematics in science» - 30.06.2014. ISJ Theoretical \& Applied Science 6(14): 44-46. - Caracas, Venezuela. doi: http://dx.doi.org/10.15863/TAS.2014.06.14.8
10. Tokmazov GV (2014) Mathematical modeling research skills in educational activity methods of probability theory. Materialy Mezhdunarodnoy nauchnoy konferenctsii "Eurapean Science and Technology" 30.11.2014. ISJ Theoretical \&Applied Science 11(20): 66-69 Southampton, United Kingdom. doi:

http://dx.doi.org/10.15863/TAS.2014.11.19.13

11. Mishhik N.A. (2016) Pravovy'e osnovy' francuzskoj si-stemy' bor'by' s zagryazneniem morya / Nauchny'e issledovaniya: Informaciya, analiz, prognoz [Tekst]: monografiya / [V.E'.Lebedev, A.A.Sviridenko, V.M.Sokolinskij i dr.]; pod obshhej red. prof. O.I.Kirikova - Kniga 51.- Voronezh-Moskva.

12. Mishchik NA (2014) The practice of french justice article 228 of the UN convention on the law of the sea. Materialy Mezhdunarodnoy nauchnoy konferenctsii "The European Science and Education"- 30.07.2014. ISJ Theoretical \& Applied Science 07 (15): 93-97. - Marseille, France. doi: http://dx.doi.org/10.15863/TAS.2014.07.15.19

13. Mishhik N.A., Antonenko G.A. (2013) Liniya gorizonta kak gradientny'j perepad V fotograficheskix izmereniyax dlya celej morexodnoj astronomii // E'kspluataciya morskogo transporta. 2013. № 2 (72). Novorossijsk, p. 23-28.

14. Mishhik N.A. (2000) Optimizaciya metodov morexodnoj astronomii [Tekst]: avto-ref.dis. ... kand. tex. nauk: 05.22.16 / N.A.Mishhik. Novorossijsk, $-24 \mathrm{p}$.

15. Mishhik N.A. (2000) Optimizaciya metodov morexodnoj astronomii [Tekst]: dis. ... kand. tex. nauk: 05.22.16 / N.A.Mishhik. Novorossijsk, $-188 \mathrm{p}$. 